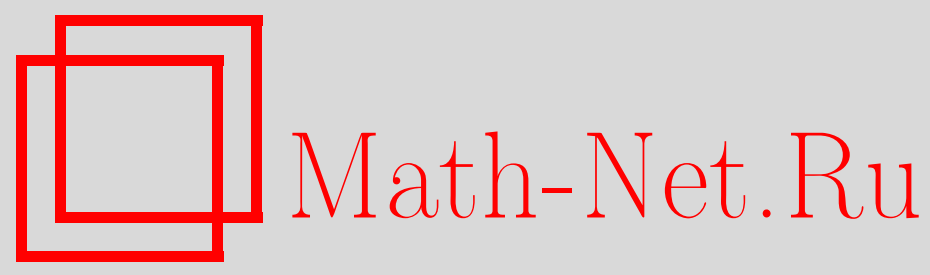

Е. А. Волков, Об одном свойстве решений уравнения Пуассона на многоугольниках, Матем. заметки, 1999, том 66, выпуск 2, 178-180

DOI: https://doi.org/10.4213/mzm1153

Использование Общероссийского математического портала Math-Net.Ru подразумевает, что вы прочитали и согласны с пользовательским соглашением http://www . mathnet.ru/rus/agreement

Параметры загрузки:

IP: 3.89 .197 .203

26 апреля 2023 г., 14:57:55

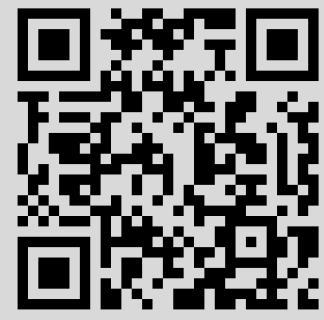




\title{
ОБ ОДНОМ СВОЙСТВЕ РЕШЕНИЙ УРАВНЕНИЯ ПУАССОНА НА МНОГОУГОЛЬНИКАХ
}

\author{
Е. А. Волков
}

Доказано, что равносторонний треугольник есть единственный многоугольник, на котором решение уравнения $\Delta u=1$ при однородных краевых условиях является алгебраическим многочленом, причем степень этого многочлена равна 3 .

Библиографоял: 2 названия.

Пусть $D$ - произвольный открытый многоугольник в плоскости $x y, \Gamma$ - его гранища. Рассмотрим задачу Дирихле

$$
\Delta u=1 \quad \text { на } D, \quad u=0 \quad \text { на } \Gamma,
$$

где $\Delta \equiv \partial^{2} / \partial x^{2}+\partial^{2} / \partial y^{2}$. Эта краевая задача, имеющая единственное классическое решение, часто возникает в приложениях (см. [1]).

ТЕорема. Равносторонний треугольник есть единственный многоугольник в плоскости ху, на котором решение краевой задачи (1) является алгебраическим многочленом, причем степень этого многочлена равна 3.

ДокАЗАтЕЛЬСтво. Допустим, что $D$ - равносторонний треугольник со сторонами, расположенньми на прямьх $y= \pm \sqrt{3} x$ и $y=h$. Тогда, очевидно, алгебраический многочлен третьей степени

$$
u(x, y)=\frac{1}{4}\left(3 x^{2}-y^{2}\right)-\frac{1}{4 h}\left(3 x^{2} y-y^{3}\right)
$$

является решением краевой задачи (1).

Установим теперь, что решение краевой задачи (1) на любом другом многоугольнике, в частности на квадрате, правильном шестиугольнике и т.д., не является алгебраическим многочленом. Остановимся на трех частных случаях.

1. Многоугольник $D$ имеет угол $\pi / 2$ или $3 \pi / 2$. Если допустить, что решение $u$ является алгебраическим многочленом, т.е. в частности, обладает непрерывными вторыми производньми вплоть до вершины рассматриваемого угла, то сразу приходим к противоречию. Действительно, сумма вторых производных, взятых вдоль сторон угла, в вершине равна 0, но $\Delta u=1$ в сколь угодно близких точках к вершине. Таким образом,

Работа выполнена при финансовой поддержке Российского фонда фундаментальых исследований, грант № 99-01-00045. 
непрерьвность хотя бы одной второй производной в вершине нарушена и, следовательно, решение задачи (1) не является алгебраическим многочленом.

2. У многоугольника $D$ имеется угол $\alpha \pi, 0<\alpha \leqslant 2$, причем либо $\alpha$ иррационально, либо $\alpha=2$. Предположим, что вершина угла находится в точке $x=0, y=0$, одна из его сторон лежит на положительной полуоси $x$, причем направление внутренней нормали к этой стороне совпадает с направлением положительной полуоси $y$. Введем полярную систему координат $\rho, \theta$ с полюсом в вершине и углом $\theta$, отсчитываемым против часовой стрелки от положительной полуоси $x$. Очевидно, существует число $\rho_{0}>0$ такое, что сектор

$$
T=\left\{(\rho, \theta): 0<\rho<\rho_{0}, 0<\theta<\alpha \pi\right\}
$$

целиком расположен в $D$, причем $\rho_{0}$ не превьшает длин сторон многоугольника, образующих рассматриваемый угол.

Представим решение $u$ задачи (1) в следующем виде:

$$
u=v+w
$$

где

$$
w=\frac{1}{2} y(y-x \operatorname{tg} \alpha \pi)
$$

- алгебраический многочлен от $x$ и $y$, удовлетворяющий уравнению $\Delta w=1$, а функция $v$ является решением задачи Дирихле

$$
\Delta v=0 \quad \text { на } D, \quad v=-w \quad \text { на } \Gamma .
$$

$\Phi$ ункция $w$ и, следовательно, гармоническая на $D$ функция $v$ обращается в 0 на сторонах сектора (3). Поэтому функция $v$ разлагается на этом секторе в ряд (см. $[2,(2.21)])$

$$
v(\rho, \theta)=\sum_{\nu=1}^{\infty} b_{\nu}\left(\frac{\rho}{\rho_{0}}\right)^{\nu / \alpha} \sin \frac{\nu \theta}{\alpha}
$$

где

$$
b_{\nu}=\frac{2}{\alpha \pi} \int_{0}^{\alpha \pi} v\left(\rho_{0}, \theta\right) \sin \frac{\nu \theta}{\alpha} d \theta, \quad \nu=1,2, \ldots
$$

Поскольку $w \not \equiv 0$ на Г и, следовательно, $v \not \equiv 0$ на $D$, то в разложении (7) имеется хотя бы один коэффициент $b_{\nu} \neq 0$; причем при $\alpha=2$ заведомо $b_{1} \neq 0$, так как в этом случае $v<0$ на всем многоугольнике $D$. Поэтому согласно теореме 2.1 [2] у функции (7) существует только конечное число производных по $\rho$ в вершине сектора, т.е. эта функция и в соответствии с (4), (5) решение $u$ задачи (1) не являются алгебраическими многочленами.

3. Многоугольник $D$ имеет угол $\alpha \pi$, расположенный так же, как и в предыдущем случае, причем $0<\alpha<2, \alpha=p / q-$ несократимая дробь, $p \geqslant 2, q \geqslant 3$. В этом случае имеем

$$
v(\rho, \theta)=\sum_{\nu=1}^{\infty} b_{\nu}\left(\frac{\rho}{\rho_{0}}\right)^{\nu q / p} \sin \frac{\nu q \theta}{p} \quad \text { на } T .
$$

Если существует коэффициент $b_{\nu} \neq 0$ для некоторого $\nu \not \equiv 0(\bmod p)$, то так же, как в предыдущем случае, устанавливается, что решение $u$ задачи (1) не является алгебраическим многочленом. 
Допустим, что $b_{\nu}=0$ для всех $\nu \not \equiv 0(\bmod p)$. Тогда

$$
v(\rho, \theta)=\sum_{k=1}^{\infty} b_{k p}\left(\frac{\rho}{\rho_{0}}\right)^{k q} \sin k q \theta \quad \text { на } T .
$$

В предположении, что $v$ является гармоническим многочленом, в (8), очевидно, может быть отлично от 0 только конечное число коэффициентов $b_{k p}$. При этом представление (8) для $v$ имеет место не только на секторе (3), но и на всем замкнутом многоугольнике $\bar{D}$.

Положим

$$
\theta^{*}= \begin{cases}\frac{\pi}{q}, & p \neq q+1, \\ \frac{2 \pi}{q}, & p=q+1 .\end{cases}
$$

Поскольку $p \geqslant 2, q \geqslant 3$, то $0<\theta^{*}<p \pi / q=\alpha \pi$, т.е. луч $\theta=\theta^{*}$ расположен внутри рассматриваемого угла многоугольника $D$, причем

$$
w\left(\rho, \theta^{*}\right) \neq 0, \quad 0<\rho<\infty,
$$

где $w$ - функция (5). В то же время,

$$
v\left(\rho, \theta^{*}\right)=0, \quad 0 \leqslant \rho<\infty,
$$

где $v$ - (по предположению) многочлен (8). Следовательно, граничное условие в (6) в некоторых окрестностях точек пересечения луча $\theta=\theta^{*}$ с $\Gamma$ не выполнено. Из полученного противоречия вытекает, что $v$ и, следовательно, решение $u$ задачи (1) не являются алгебраическими многочленами.

Завершим доказательство теоремы. В пунктах 1-3 в совокупности доказано, что если у многоугольника $D$ имеется хотя бы один угол $\alpha \pi \notin\{\pi / q: q \geqslant 3\}$, то решение $u$ краевой задачи (1) не является алгебраическим многочленом. Поэтому, для того чтобы решение задачи (1) на многоугольнике $D$ было алгебраическим многочленом, необходимо наличие у этого многоугольника углов только вида $\pi / q$, где $q \geqslant 3$ - некоторое целое. Таким многоугольником является только равносторонний треугольник, на котором найденное решение задачи Дирихле (1) имеет вид (2), отвечаюший утверждению теоремы.

Теорема полностью доказана.

\section{СПИСОК ЦИТИРОВАННОЙ ЛИТЕРАТУРЫ}

[1] Седов Л. И. Механика сплошной среды. Т. 2. М.: Наука, 1994.

[2] Волков Е.А. О дифференциальных свойствах решений краевых задач для уравнения Лапласа на многоугольниках // Тр. МИАН. 1965. Т. 77. С. 113-142. 